\title{
Para un examen crítico del comparatismo francés ${ }^{\circ}$
}

\author{
Michel Riaudel ${ }^{\bullet}$ \\ CRIMIC, Sorbonne Université \\ Traducción del francés: \\ Silvia Zenarruza de Clément ${ }^{\bullet \bullet}$ \\ Universidad Nacional del Litoral
}

\section{Resumen}

La ausencia de la literatura brasileńa en los programas de literatura comparada de los concursos de reclutamiento de docentes en Letras modernas es el indicio del achicamiento de la disciplina a favor del trípode franco-anglo-germánico. Si una mayor apertura al mundo es necesaria, es sin embargo delicado establecer umbrales de justas representaciones de las literaturas extranjeras. La cuestión remite, de hecho, a la constitución del valor literario, y pone de manifiesto también el carácter algo oblicuo de estudios efectuados en un marco estrictamente nacional. Es por eso que, paradójicamente, el hecho que los comparatistas inscriban en su campo la literatura brasileña y otros dominios olvidados podría enseñar a relativizar el pedestal nacional de los estudios literarios.

\section{Palabras clave}

· Literatura brasileña · literaturas extranjeras · comparatismo

\footnotetext{
- Este texto fue leído el 22 de julio de 2013 en el ciclo «Le comparatisme France / Brésil: un bilan critique», organizado por el Centre «Littérature et Poétique Comparées» (Université Paris Ouest - Nanterre), partner del 20e Congrès de l'Association Internationale de Littérature Comparée.

- Dr. en Literaturas Comparadas. Profesor de la Sorbonne Universitè; miembro titular del Centre de Recherches Interdisciplinaires sur les Mondes Ibériques Contemporains (CRIMIC). Responsable del Centro de Estudios Portugueses del CRIMIC y de la Sección de Estudios Lusófonos del UFR de estudios ibéricos y latinoamericanos. Sus intereses de investigación se relacionan con la difusión literaria entre Brasil y Francia, literatura brasilera y cuestiones relacionadas a intertextualidad, traducción, la relación entre literatura y ciencias sociales, entre otras

-.- Licenciada en Literatura y Lenguas Modernas, Profesora en Letras, Profesora de Francés, Profesora adjunta ordinaria, integrante del equipo de cátedra Literatura Francesa e Italiana de la Facultad de Humanidades y Ciencias Universidad Nacional del Litoral. Profesora (adjunta) de francés en la Facultad de Ingeniería Química de la UNL. Profesora en el ISP No 8 A. Brown. Directora de la Alianza Francesa de Santa Fe. Autora de numerosos artículos. Integra el equipo de investigación dirigido por la titular Adriana Crolla.
} 


\begin{abstract}
Brazilian literature is notoriously absent from the syllabi contents of exams for Modern Languages teachers aspiring to find employment. This clearly indicates a reduction in the scope of the discipline, thus favouring the traditional Franco-Anglo-German spectrum. We gather that it is necessary to embrace a worldwide perspective and establish a threshold that should more proportionally represent foreign literatures. This clearly implies a consideration of what constitutes literary value and also shows a certain degree of bias in studies carried out under a strict national frame of mind. Thus, including Brazilian texts —as well as other ignored writingsin Comparative Literature Studies would help to bring the national pedestal of literature to a more accurate perspective.
\end{abstract}

\title{
Key words
}

Brazilian literature $\cdot$ foreign literatures · comparatism

\author{
"La reflexión nace de ideas comparadas y es la pluralidad de ideas \\ lo que lleva a compararlas. Aquél que no ve más que un solo objeto \\ no tiene ninguna comparación para hacer. Aquél que no ve más \\ que un pequeño número de ideas, y siempre las mismas desde su infancia, \\ no las compara tampoco, porque el hábito de verlas le quita la atención necesaria \\ para examinarlas: pero a medida que un objeto nuevo nos choca, \\ queremos conocerlo; le buscamos una relación con aquellos que conocemos. \\ Es asi que aprendemos a considerar el examen de lo que nos emociona" \\ JeAn-Jacques, Rousseau, Ensayo sobre el origen de las lenguas, \\ donde se habla de la Melodía y de la Imitación musical, 1781, cap. IX.
}

\section{Cartografía}

En 1994, según los datos del Sindicato nacional de la edición, se tradujeron 1347 títulos venidos de los Estados Unidos y de Gran Bretaña, 89 de lengua alemana, 58 de español, 14 de portugués ${ }^{1}$. O sea, una relación de 1 a 100 entre la introducción anglosajona y la introducción de los espacios lusófonos. Se podría poner en relación esta constatación con la enseñanza del portugués en el secundario, que conoció una notable progresión en los años $1970^{2}$ para disminuir progresivamente dos décadas más tarde. En una interdependencia comprensible, los estudios portugueses y brasileños están entre dos fuegos: por una parte un retroceso de la enseńanza de especialidad, corolario de la rareza (o de la ausencia) de puestos en los concursos (CAPES, Agregación); 
por otro de una demanda constante y creciente de formaciones complementarias por parte de los no especialistas. Muchas universidades han debido cerrar su Licencia LLCE y su máster de especialidad de literaturas de expresión lusófona, mientras que docentes de portugués deben a veces rechazar inscripciones en las UE de iniciación, libres o menores, por falta de lugar.

Una parte de la enseñanza superior francesa y algunas grandes escuelas tienen conciencia de los desafíos de una cooperación con Brasil o con las antiguas colonias africanas de Portugal, cuyo desarrollo pasa sobre todo por el reclutamiento de especialistas de esas áreas geo-lingüísticas. La Universidad de Poitiers ha creado un «recorrido internacional», horario reforzado de aprendizaje de la lengua portuguesa, rusa o china, y de sus culturas, propuesto sobre los seis semestres de licencia, a estudiantes de todas las carreras relacionadas. Pero en una atmósfera donde el oxígeno se agota, la cuestión de la renovación del plantel docente formado por la Universidad, de traductores, de especialistas capaces de intervenir en diversos niveles, se plantea ${ }^{3}$.

¿Qué ocurre con el interés del comparatismo francés para el campo brasileño? Cuando se intenta un electrocardiograma de la disciplina a través de las obras puestas en el programa de la agregación de letras modernas estos últimos veinte años ${ }^{4}$, se constata que nueve obras sobre diez son europeas (Figura 1). Además, la Europa central o anglófona pesa - tanto una como la otra— separadamente, más que sus márgenes del norte, del sur y del este reunidos (Figura 2). Siempre bajo el ángulo geopolítico, la suma de los autores americanos (anglófonos e hispanófonos) cuenta menos del 8\%. Un turco, Nazim Hikmet, vale por todo el Oriente, el Asia. En esta carta del mundo, dos continentes no existen.

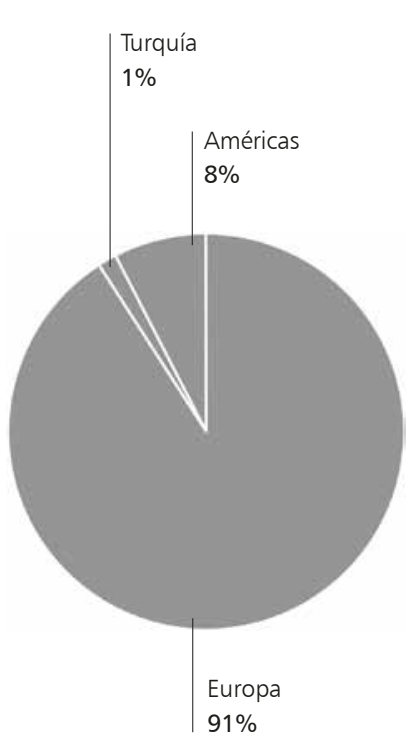

Figura 1. Origen continental de las obras en los programas de la prueba de literaturas comparadas. Agregación de Letras Modernas (1993-2014).

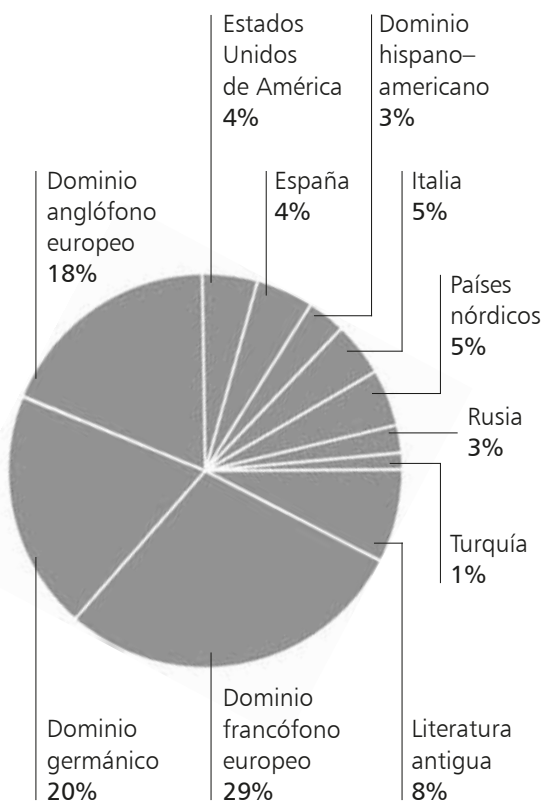

Figura 2. Origen regional de las obras en los programas de la prueba de literaturas comparadas. Agregación de Letras Modernas (1993-2014). 
Desde el punto de vista lingüístico, junto al dominio francófono que representa cerca del 30\% de los autores, el conjunto anglófono, uniendo a todos los continentes, domina ligeramente al bloque germánico, cada uno constituyente del orden de una quinta parte de los programas. Pero la relación se invierte si sólo se considera el conjunto británico, fuera de los Estados Unidos, lo que señala una sobre representación alemana (Figuras 3 y 4). Por otra parte la diversidad del repertorio germánico es muy superior a la del corpus británico, donde aparece seis veces el nombre de Shakespeare. De todas maneras, tres lenguas se reparten cerca de la tres cuarta parte de las obras de los programas (Figura 4) en las que no se nota ninguna presencia lusófona. Difícil de precisar cuándo un autor brasileño ha sido inscrito una vez hace mucho tiempo.

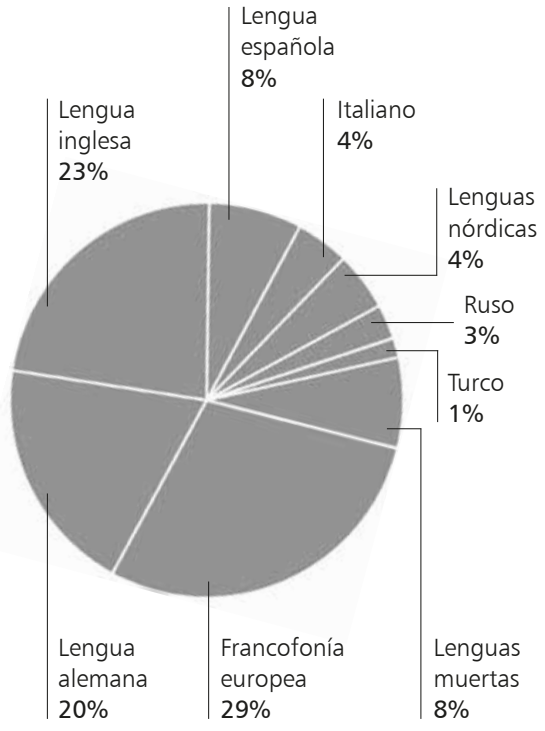

Figura 3. Áreas lingüísticas de las obras en los programas de la prueba de literaturas comparadas. Agregación de Letras Modernas (1993-2014).

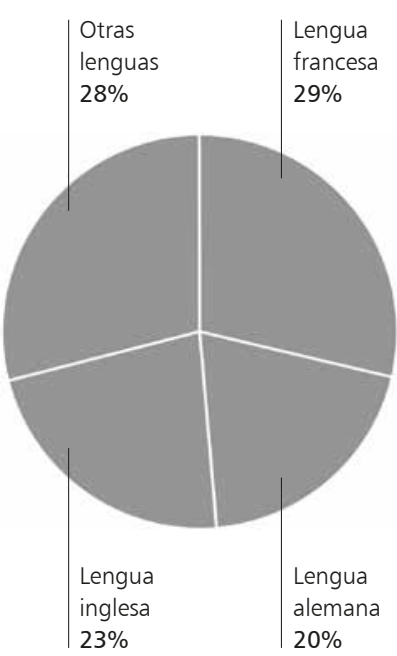

Figura 4. Lenguas de las obras inscriptas en los programas de la prueba de literaturas comparadas. Agregación de Letras Modernas (1993-2014).

Tal es el filtro a través del cual se reproducen las fuerzas vivas de la literatura comparada francesa. Si su terreno lingüístico o geopolítico de todos los días no se reduce por cierto a ese pasaje estrecho, ni mucho menos, el panorama que surge de los programas de los concursos infiere una producción científica de circunstancia que refuerza sus áreas de especialización (corpus y lenguas). Los programas de concurso ilustran un sistema, con sus mecanismos endogámicos perversos, por donde una disciplina se reproduce y produce acentuando implacablemente los mismos tropismos. Se concibe que una corporación cuyo centro de gravedad se sitúa entre el francés, el alemán y el inglés tendrá tendencia a inscribir en los programas de concurso obras que ella conoce, sobre las que ya existe una bibliografía que ella sabrá enriquecer y que servirá de criba para seleccionar el relevo. Este eurocentrismo del comparatismo oficial francés hace que no pueda pretender ocupar a este respecto ni la posición mediana entre el norte y el sur que reivindicaba Edgar Quinet, ni (menos aún) un lugar universal. 


\section{Contextos}

La recepción brasileña en Francia, no más que la del sur europeo, no ha tenido siempre ese carácter de rareza que ilustra, desde otro punto de vista, la suerte de los trabajos pioneros de Pierre Rivas, sobre el triángulo literario entre Francia, Brasil y Portugal: no han retenido mucho la atención de los brasileños, que los han reunido, traducido y publicado 5 . Sin remontar a la época de Ferdinand Denis, autor de la primera historia literaria del Brasil mientras que la nación tenía apenas cuatro años de existencia ${ }^{6}$, recordemos que las literaturas iberoamericanas han sido tratadas con alguna consideración durante más de un siglo. En 1846 era creada en el Collège de France la «Cátedra de Lenguas y Literaturas de la Europa Meridional», asumida por Edgar Quinet. Le sucedieron Alfred Morel-Fatio, luego el comparatista Paul Hazard (1925-1944), cuya entronización fue acompañada por un cambio de definición: "Cátedra de Historia de las literaturas comparadas de Europa Meridional y de América Latina». Es gracias a él, por otra parte, como lo han hecho notar Daniel-Henri Pageaux ${ }^{7}$ y Tânia Carvalhal ${ }^{8}$, que la Revue de littérature comparée se abriría en Brasil seis años después de su fundación, con un artículo titulado: «Del Antiguo al Nuevo Mundo: los orígenes del romanticismo en el Brasil»». Fuera del dossier sobre América Latina de 1931 y el que trataba exclusivamente del Brasil, en $2005^{10}$, es decir oportunamente concomitante con el Año del Brasil en Francia y de la presidencia de la Asociación Internacional de Literatura Comparada por una brasileña, dos años antes de la realización del congreso mundial de la AILC en Río de Janeiro, la presencia luso-americana en la RLC quedó y sigue siendo muy accidental. La apertura, aprovechando el Internet latinoamericano que se intensificó después de la Segunda Guerra Mundial ${ }^{11}$, nunca fue relanzada más que parcialmente, en ocasión de celebraciones o de circunstancias efímeras.

En el frente editorial hubo también épocas más felices. Es cierto que los brasileristas franceses de antes de 1945,

[...] formados por el azar (actividad comercial, por ejemplo, en el caso de Duriau) y en una estética tradicional, no eran muy sensibles a la modernidad, en busca de un Brasil más pintoresco que de avanzada, más regional o regionalista, que cosmopolita, más próximo del cuento etnográfico que de la poesía modernista. (Rivas, 1996: 315) ${ }^{12}$

Pero el viento del tercermundismo y del cosmopolitismo que sopló entre 1945 y los años 1970 nos aportó numerosas traducciones, incluyendo la poesía. La colección Seghers acogió a la generación de 1945, reservándole una parte proporcionalmente más bella que a los modernistas: se encuentran allí los nombres de Vinicius de Moraes $^{13}$, Murilo Mendes ${ }^{14}$, Manuel Bandeira ${ }^{15}$, Cecília Meireles ${ }^{16}$, mientras que Gerardo Mello Mourão era editado en Gallimard ${ }^{17}$, y Carlos Drummond de Andrade en la colección bilingüe de Aubier ${ }^{18}$. El mismo público pudo simultáneamente des- 
cubrir las primeras traducciones de Clarice Lispector ${ }^{19} \mathrm{y}$ de João Guimarães Rosa ${ }^{20}$. La nueva especialización, señalada por la pionera colección de literatura brasileńa de las ediciones Métailié, es contemporánea de la antología dedicada a Oswald de Andrade o de la traducción de Macunaíma de Mário de Andrade ${ }^{21}$; ésta encontró su traducción diplomático-cultural con la operación «Bellas extranjeras» de 1987, que reunía en París a una treintena de escritores brasileños. Después de una puesta al día fecunda operada durante los ańos 1980-1990, las traducciones francesas de la literatura brasilera de nuevo se espaciaron y carecen singularmente de visibilidad.

Se podría sugerir que el contexto ha cambiado hoy, que el bloque latinoamericano no es más políticamente capital como lo fue en los años 1960-1970, cuando relevaba las luchas de descolonización con Cuba y la eclosión de los movimientos post-guevaristas, o cuando movilizaba una opinión contra la diseminación de regímenes dictatoriales particularmente sanguinarios, dando una dimensión NorteSur al conflicto Este-Oeste. Pero la observación supone que la audiencia literaria sería antes que nada todo factor de relaciones geopolíticas y que el trabajo crítico sería menos la ocasión de actuar para hacer pensar y revisar representaciones, que un reflejo de las convenciones instaladas y de las coyunturas históricas. Hipótesis éstas que, si se confirmasen, harían del comparatismo un relevamiento de conformidades políticas o un lugar de reproducción de lo mismo.

\section{Focales}

A esta perplejidad quisiéramos agregar tres niveles de consideraciones problemáticas sobre la apreciación de una recepción nacional: ¡cuáles son las medidas confiables? ¿Cuándo se puede hablar de sub-representación de una literatura nacional? ¿El marco nacional es el más apropiado para evaluar una recepción?

Con respecto a los gradientes, hay que insistir en su valor relativo. Un número de intraducciones no tiene sentido sino comparado con cifras globales, comparado a otros datos nacionales o regionales, así como a los mismos elementos establecidos por períodos diferentes. Así, la progresión de las traducciones de la literatura brasileña, incontestable en valor absoluto en el curso del siglo XX (una por año al principio del siglo, una media de veinticinco títulos anuales en el último decenio), no puede interpretarse sino relacionada con el volumen de todas las traducciones en el mercado francés: se publica mucho más que en 1900, y los editores son alentados a traducir por subvenciones y mecanismo del oficio ${ }^{22}$.

Por otra parte la clasificación propuesta por Gisèle Sapiro, distinguiendo la situación hiper-central de una lengua, contra las centrales, semi-periféricas y periféricas, de las otras, si bien es muy útil, introduce distorsiones insidiosas: focalizándose en las dominaciones, mira en sentido único, allí donde sería útil pensar en términos de "polisistemas" que se encajan unos en otros o se solapan. Ubicar por ejemplo el chino en las lenguas periféricas tiene un sentido en la escala mundial, pero borra la percepción de la hegemonía que puede ejercer regionalmente. Si el español es una lengua semi-periférica y el portugués una lengua periférica vista la tasa de intraducciones planetarias, son sin embargo ellos dos la llave de América Latina. Sin ellos, un semi-continente (al menos) desaparecería de la superficie del globo, como una nueva Atlántida tragada por los efectos de escala. 
La sistematización de las interpretaciones recorta la realidad, la alisa y termina construyendo otra: muchos creen que el inglés es una suerte de pasaporte cuando no abre más que a una delgada capa superficial de mundos, frecuentemente en un registro de escasas nociones. Esta concepción instrumental de la comunicación razona en una banda de intercomprensión reducida: el globish no ayuda a la lectura de los poetas metafísicos o de Faulkner. El anverso de la hiper-centralidad del inglés es además que resulta poco eficaz en los estudios muy especializados de dominios extranjeros: la bibliografía anglófona sobre la literatura brasileńa existe pero no es para nada un sésamo. No conocer el portugués es, por el contrario prohibirse el conocimiento de la literatura de Portugal, del Brasil, de Angola, de Mozambique, etc., como no conocer el danés excluye los estudios de la literatura danesa, aún si el inglés permite sobrevivir en ese país.

El enfoque cuantitativo de la recepción choca además con otro problema de interpretación, que una cuestión a la vez de sentido común y extravagante ayudará a percibir: puesto que se trata aquí o allá de sub-representación o de sobre-representación de una literatura nacional, ¿en qué podría consistir su representación justa? ¿Estaría en adecuación con la parte de PIB en el producto mundial? ¿El peso demográfico? ¿La superficie del territorio correspondiente? ¿La densidad de su población? ¿La influencia geopolítica? ¿El lugar en las instituciones nacionales? Si estos criterios pueden parecer insólitos en el marco de un estudio literario, reposan sobre la evidencia que no se podrá (no se debería) esperar el mismo volumen de intraducciones provenientes de un país de un poco más de sesenta millones de habitantes o el de aquél proveniente de una nación que pasa los mil millones de ciudadanos. Más aún, ponen a la luz que estas consideraciones sobre circulaciones literarias equitativas tienen resortes más políticos, sociales, identitarios o estratégicos que estéticos. La idea de déficit no tiene sentido sino relacionada con una situación juzgada equilibrada, imposible de determinar puesto que implica lo imaginario, la percepción individual, el punto de vista, la toma de posición. No hay una situación normal, lo mismo que no existe un instrumento ideal estableciendo el nivel aceptable de una recepción, sino sólo herramientas aproximativas. Todo es, en este campo, distorsión y asimetría - la asimetría no es reductible a una situación de dominación, a juegos de poder... Digamos que existen, a pesar de todo, asimetrías más fecundas que otras, así como existen actitudes críticas frente a esas distorsiones más fecundas que otras. Es por eso que a lo uniforme preferimos lo diverso.

De cualquier manera, tenemos que renunciar a la hipótesis de una correlación mecánica entre cantidad y calidad. Ni el número de extraducciones de una obra, ni su cifra de ventas son una garantía de valor literario, puesto que hay que distinguir un éxito coyuntural de un éxito de larga duración. Existe, sin embargo, entre ellas una relativa y compleja imbricación, que desmiente la creencia que un gran libro terminará siempre por encontrar a su público. Ahora bien, en el mundo de las letras todas las obras nacen teóricamente libres e iguales en derechos, pero en la práctica, algunas tienen chances de reconocimiento más iguales que otras. $\mathrm{Si}$ se considera, por una parte, que la obra literaria está destinada a circular $^{23}$; y, por otra parte, que el valor literario de esta obra puede precisamente medirse en su expansión, su capacidad en suscitar la lectura y la reapropiación, y hasta cambiar nuestra visión sobre el mundo; entonces concluiremos que este valor de uso o de cambio está más o menos considerablemente obstaculizado (o facilitado), aún sobre el plano estético, en función de las redes de circulación en las que se inserta. 
La lectura de un libro no depende únicamente del genio de un autor que encuentra la buena disposición de un adquiriente. Su historia está hecha por un mixto de azar, de condiciones sociales y de clinamens individuales capaces de producir un «efecto de transfert». De manera que está, entre otros factores, subjetivamente $e^{24}$ sobredeterminado por los múltiples agentes de la circulación. Es en esto que es necesario, desde este punto de vista, invertir la dicotomía entre lo político y lo estético a partir de la cual hemos razonado hasta ahora, y afirmar que la promoción de la literatura brasileña pasará sobre todo por la acción pública, que surgirá de una voluntad política ejercida tanto a nivel de un gobierno como del de las mediaciones culturales, de las instancias universitarias o de las sociedades intelectuales. Para ensanchar esta tesis, se podría reformularla cambiando la célebre meditación XVII de John Donne:

[Ninguna obra] es una isla, completa en ella misma; [cada una] es un trozo del continente, una parte del conjunto; si un pedazo de tierra es acarreado por el mar, Europa será menoscabada, como lo estaría un promontorio, como lo estaría la mansión de tus amigos o la tuya ${ }^{25}$.

Privarse del corpus brasileño es menoscabar el dominio de la literatura. De hecho, en otra interpretación, esta paráfrasis sugiere a la vez la inadecuación del marco nacional para pensar la literatura y la imposibilidad de salir de ella. Hablar de literatura nacional es en efecto predeterminar las obras en virtud de una incompletud —la nación figura allí como una totalidad superior a la de las obras tomadas individualmente- y a partir de una entidad histórica con la cual no coinciden siempre. La Grecia de la Odisea no se puede superponer a la nación contemporánea que tiene como capital Atenas; el Brasil de Gregório de Matos no puede ser confundido con el país que se independizó en 1822. Antônio Vieira ¿corresponde a la historia literaria brasileira, portuguesa, luso-brasileira? ¿Dónde situar los mitos amerindios? ¿La producción de los viajeros? Desde el punto de vista brasileño, ¿jean de Léry no es también tan extranjero como Pero Vaz de Caminha? Se ve aquí que se mezclan los criterios políticos, jurídicos, lingüísticos en la determinación nacional de una obra, lo mismo que se solapan las categorías históricas (sin embargo inconfundibles) de "poesía» y de «literatura» en la constitución de un canon. De ese marco nacional, a menudo efecto de una retroyección anacrónica, animada por una teleología particularmente activa en los estudios de literatura colonial confundiendo el sentimiento nativista y la reivindicación de pertenencia ligada a la realidad del Estado-Nación, resultan numerosas investigaciones críticas apuntando a identificar tal o cual rasgo identitario en obras de hecho extranjeras al proyecto de nación que se les supone. El marco nacional de los estudios literarios induce una expectativa que encierra y somete las conclusiones críticas, a la que tendrá que reducir los escritos, ya fueran de Clarice Lispector o de João Guimarães Rosa, suponiendo entre ellos un continuum y una connivencia que no tienen más que con los de Goethe o de Mansfield.

Sin embargo, a aquellos que consideran, con buenos o malos argumentos, que la dimensión nacional está envejecida y es regresiva, fosilizada en los siglos XIX y XX durante los cuales su invocación produjo las calamidades conocidas, o que está superada, laminada por la globalización, responderemos que su denegación pretende transformar el enraizamiento en hecho indiferente, transparente, en el momento mismo en que ella lo sobrevalora en la práctica, como lo demuestran 
empíricamente los gráficos que preceden. A decir verdad, la Nación es hoy más que nunca una dimensión central de nuestro planeta globalizado, en la medida en que la globalización (como en otra época la colonización) necesita de la existencia de los Estados: tiene, a su manera, necesidad de fronteras y de particularismos que mantiene y alimenta al mismo tiempo que finge superarlos ${ }^{26}$.

Al final de cuentas, uno podría preguntarse por qué habría que leer, entre todos los particulares posibles, la literatura brasileña. Más allá de las obras maestras que no hay que enumerar aquí, nos contentaremos con una respuesta en dos tiempos, cada uno con su forma de inversión. Primero, precisamente, no porque ella sería más digna de ser estudiada que otra - como tampoco sería legítimo ignorarla si lo fuera menos que otra. Hay que interesarse en ella, en suma, no en nombre de comparativos, o de superlativos, sino de la comparación, razón de ser de una disciplina, la literatura comparada, una de cuyas virtudes es la de desplazarnos en nosotros mismos. Comparar es salir del solipsismo, tanto para comprender al otro como para comprenderse a sí mismo a través del otro. Y si la literatura comparada se declara igualmente general, si se ocupa de cuestiones trascendiendo la dimensión nacional, no puede hacerlo sino tomando en cuenta el terreno empírico de las lenguas y de las culturas. No llegaremos a una literatura comparada arcoiris haciendo abstracción de los colores. El comparatismo francés sufre de un biés francés, la bipolarización en torno a los términos «universal/comunitario», que deconstruyen felizmente reflexiones como, entre otros, las de Jacques Derrida ${ }^{27} \mathrm{o}$ las propuestas sobre la noción de común del sinólogo François Jullien ${ }^{28}$. La literatura brasileña las desarrolla o las ilustra a su manera.

Aquí, por otra parte, reside el segundo motivo de alegato para el estudio de las circulaciones franco-brasileñas en dirección del comparatismo francés: como una suerte de desafío contradictorio, provee uno de los mejores antídotos al marco nacional de los estudios literarios. Es ante todo la escuela de los malentendidos: las incomprensiones y equívocos, entre Blaise Cendrars y Oswald de Andrade, Benjamin Péret y los modernistas..., jalonan mucho más su historia que los encuentros felices, y revela las aleas fructuosas del transfert. Esta desmitificación del diálogo entre culturas, tal como está idealizado por ingenuidad o cálculo diplomático, lejos de concluir en su inexistencia, nos enseńa que no es lo que se cree o pretende que es. La segunda lección, especie de despliegue de la primera, conduce a repensar la relación del uno al otro: de la fusión a la disociacióm, de las apariencias al falso parecido, pasando por la resistencia y el contrapunto... Es toda la cuestión de la relación colonial, como de la independencia, de la alienación y de los modelos que se encuentra inscripta en los estudios sobre el barroco, en la ilusoria continuidad de los romanticismos transatlánticos, en la provocación de la antropofagia cuya fórmula matemática podría ser $2=1=3$. Nada más eficaz que esos terrenos, tomados en serio, para salir de la perspectiva organicista o biológica de la historia literaria. Sin contar que una vuelta por el Brasil debería curar a más de uno de su sentimiento de superioridad ante pretendidos discípulos. En fin, tercera enseñanza posible, entre muchas otras: integrar el repertorio brasileño conduce a relativizar las rigideces nacionales cuestionando el fundamento mismo de sus límites: ¿derecho de suelo? ¿derecho de sangre? ¿sistema? Esos debates, que atraviesan la crítica brasileña, no deben ser importados como tales, sino que valen por su inspiración problemática. Así actuará tal vez la excursión brasileña, desinflando la plena suficiencia europea: revelarnos lo que nos falta. 
Notas

${ }^{1}$ Datos establecidos sobre las compras de derechos de traducción.

${ }^{2}$ Creación del diploma CAPES de portugués en 1970, de la agregación en 1974

${ }^{3}$ Según el informe de Olinda Kleiman, $20 \%$ de los docentes del secundario eran interinos o contratados en 2011: «Situación actual de la enseñanza del portugués en la universidad francesa», en CHristian Lagarde y Philippe Ragaté (dir.), HispanismoS, n 2: «Transversalidad y visibilidad disciplinarias: los nuevos desafíos del hispanismo», junio 2013, p. 39. Disponible en http://www.hispanistes.org/images/PDF/ HispanismeS\%20n2\%20Olinda\%20Kleiman.pdf (última consulta el 12 de septiembre de 2013).

${ }^{4}$ Hemos censado los programas de la prueba de literatura comparada en la agregación de Letras modernas desde 1993 hasta 2014. Sólo nos han faltado los datos de 1999.

${ }^{5}$ Pierre Rivas, Encontro entre literaturas. França-Brasil-Portugal, trad. coordinada por Durval Ártico e Maria Letícia Guedes Alcoforado, São Paulo: Hucitec, 1995; et Pierre Rivas, Diálogos interculturais, São Paulo : Hucitec, 2005.

Se notará en esta misma lógica que el primer volumen de l'Histoire des traductions en langue française. Dix-neuvième siècle (1815-1914), notable obra dirigida por Yves Chevrel, Lieven D’Hulst y Christine Lombez (Paris: Verdier, 2912), ignora pura y simplemente las traducciones del dominio brasileńo (Eugène de Monglave sobre todo), tanto como el papel de pionero de Ferdinand Denis, a pesar de su vocación exhaustiva. ${ }^{6}$ Ferdinand Denis, Résumé de l'histoire littéraire du Portugal [seguido de] Résumé de l'histoire littéraire du Brésil, Paris: Lecointe et Durey Libraires, 1826. La independencia brasilera ha sido proclamada en 1822.

${ }^{7}$ Daniel-Henri Pageaux, Trente Essais de Littérature Générale et Comparée ou la corne d'Amalthée, Paris: L'Harmattan, 2003, p. 93.

${ }^{8}$ TÂNia Carvalhal, "Le Brésil dans la $R L C$ ", in Revue de littérature comparée, Paris: Klincksieck, n 316, 2005/4, p. 399.

${ }^{9}$ Revue de littérature comparée, Paris, 1927, pp. 111-128.

${ }^{10}$ Hay que señalar que las contribuciones provienen en su mayor parte del mundo sudamericano, lo que confirma la pobreza de la fuente francesa. ${ }^{11}$ Así el 4 de diciembre de 1945, Marcel Bataillon era promovido a la nueva cátedra del Collège de France de Lenguas y Literaturas de la Península Ibérica y de América Latina.

${ }^{12}$ Pierre Rivas, «Réception critique de Macounaïma en France». En Mário de Andrade, Macounä̈ma, edición crítica dirigida por Pierre Rivas, trad. Jacques Thiériot, Paris: Stock, ALLCA XX, CNRS, 1996, p. 315. « [...] formados ao acaso (atividade comercial, por exemplo, no caso de Duriau) e numa estética tradicional, eles não eram muito sensíveis à modernidade, preocupados com um Brasil mais pitoresco que vanguardista, mais regional, e até mesmo regionalista, que cosmopolita, mais próximo do conte etnográfico do que da poesia modernista», Pierre Rivas, "Recepção e crítica de Macunaima na França», in Pierre Rivas, Diálogos interculturais, op. cit., p. 90. 
${ }^{13}$ Cinq élégies, trad. Jean-Georges Rueff, Paris Seghers, 1953 ; Recette de femme et autres poèmes, trad. Jean-Georges Rueff, Paris : Seghers, 1960. ${ }^{14} \mathrm{Su}$ expatriación convierte en verdad su recepción europea en atípica: Murilo Mendes, Office humain, trad. Dominique Braya \& Saudade Cortesăo [la mujer del poeta], Seghers, 1956.

${ }^{15}$ Manuel Bandeira, Poèmes, trad. Manuel Bandeira, Aníbal Falcão, F.H. Blank-Simon, Paris: Seghers, 1960 (una selección hecha por el autor); Manuel Bandeira, estudio, elección de los textos y bibliografía por Michel Simon, Paris: Seghers, 1965.

${ }^{16}$ Cecília Meireles, Poésies, trad. Gisèle Slesinger Tygel, Paris: Seghers, 1967. Estas ediciones están hoy casi todas indisponibles.

${ }^{17}$ Le valet de pique, trad. Wanda Pénicaut et Violante do Canto, Paris: Gallimard, 1966.

${ }^{18}$ Carlos Drummond de Andrade, Réunion/Reunião, éd. bilingüe, trad. Jean-Michel Massa, Paris: Aubier-Montaigne, 1973.

${ }^{19}$ Clarice Lispector, Près du coeur sauvage, trad. Denise Teresa Moutonnier, Paris: Plon, 1954.

${ }^{20}$ JoÃo Guimarães Rosa, Buriti, trad. Jean-Jacques Villard, Paris: Seuil, 1961 [original: JoÃo Guimarães Rosa, «Dão-lalaláo», «O recado do morro", "Uma estória de amor», in Corpo de baile, 1956]. Ver en este campo la tesis de doctorado de Márcia Valéria Martinez de Aguiar, «Traduzir é muito perigoso: as duas versōes francesas de Grande sertão: veredas - historicidade e ritmo» (São Paulo, Études linguistiques, littéraires et traductologiques en français, Département de Lettres modernes, USPFFLCH, 2010, sous la direction de Mário Laranjeira). Esta investigadora trabaja actualmente en el marco de una investigación post-doctoral, en la edición crítica de la correspondencia entre João Guimarães Rosa y Jean-Jacques Villard, así como en el contexto de recepción francés de esta obra en los años sesenta. Sus conclusiones muy esperadas parecen confirmar esta efervescencia de América Latina y, en cuanto a lo que nos interesa, del Brasil, aunque la historia literaria no haya retenido finalmente más que el éxito del boom hispanoamericano.

${ }^{21}$ Mário de Andrade, Macounaïma le héros sans aucun caractère, trad.

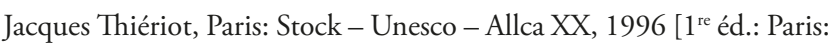
Flammarion, 1979]. Oswald de Andrade, Anthropophagies. Mémoires sentimentaux de Janot Miramar, Séraphin Grand-Pont; Manifeste de la Poésie Bois Brésil; Manifestes et textes anthropophages, trad. Jacques Thiériot, Paris: Flammarion, 1982.

${ }^{22}$ Este sistema de preventa virtual de los libros ubicados en librerías asegura una entrada artificial de tesorería a los editores que se benefician con ello, pronto compensada por otra ola de títulos.

${ }^{23}$ Ver sobre este punto, nuestro texto: «Réflexions sur l'étude des transferts littéraires», en Anaïs Fléchet et Marie-Françoise LéVy (dir.), Actas del colloque «Les circulations littéraires et musicales au $\mathrm{XX}^{\mathrm{e}}$ siècle» (14 et 15 mars 2013, Université Paris 1 - UMR-IRICE - Université de Versailles Saint Quentin en Yvelines). En prensa.

${ }^{24}$ Subjetivamente, y no objetivamente, en la medida misma en que, en una paradoja sólo aparente, el elemento económico (sobre el cual buscan 
actuar por ejemplo los programas de ayuda a la traducción) tienen más que nada un componente subjetivo que condiciona el recurso al efecto de ganga. Aquí como en otra parte, la creencia juega un rol esencial.

${ }^{25}$ John Donne, Méditations en état de crise, traducido y con prefacio de Franck Lemonde, Paris: Payot, Rivages poche-Petite Bibliothèque, 2002, p. 71-73.

${ }^{26}$ Económicamente, dicha globalización sabe muy bien explotar las diferencias fiscales, de remuneración o de derecho del trabajo entre los países. ¿Cómo haría sin Suiza, Luxemburgo, la China o la India? Podría ser, simplemente, un avatar de la lógica colonialista, que tomaría el control de territorios y de pueblos en nombre de una universalidad, religiosa, científica o republicana, mientras que se aplicara a fragmentarla en zonas de primera o segunda pertenencia. Uno de los matices con el mundo de ayer reside tal vez, sin embargo, en los descentramientos de los lugares de poder y de decisión, que no se puede identificar pura y simplemente con los Estados.

${ }^{27}$ Ver, en particular, JACQUes DeRrIDA, Le monolinguisme de l'autre ou la prothèse d'origine, Paris: Galilée, coll. «Incises», 1996.

${ }^{28}$ François Jullien, De l'universel, de l'uniforme, du commun et du dialogue entre les cultures, Paris: Fayard, 2008 (retomado en Points-Seuil). 